\title{
Efficacy and Safety of Docetaxel or Epirubicin, Combined with Cisplatin and Fluorouracil, (DCF and ECF) Regimens as First Line Chemotherapy for Advanced Gastric Cancer: a Retrospective Analysis from Turkey
}

\author{
Fatih Teker*, Bahiddin Yilmaz, Yasemin Kemal, Engin Kut, Idris Yucel
}

\begin{abstract}
Objectives: Advanced gastric cancer (AGC) patients have a poor prognosis. The best benefit of chemotherapy is usually achieved by first line setting. Very few studies have compared combination regimens. This study was designed to compare two combination regimens. Methods: Patients with advanced gastric cancer receiving first line chemotherapy were retrospectively collected, and divided into two groups, receiving DCF (docetaxel, cisplatin and fluorouracil) or ECF (epirubicin, cisplatin and fluorouracil) regimens. Data were collected for the retrospective analysis in a single center. Results: Eighty-six patients were eligible for analysis. Median overall survival (OS) was 10.0 months in the ECF group and 11.0 months in the DCF group (p=0.31). Median progression free survival (PFS) for ECF and DCF was equal at 6.0 months. Second line chemotherapy were administered in more than one third of patients. Both regimens had similar toxicity. Conclusions: This is the first study investigating the outcomes of gastric cancer chemotherapy in this region. ECF and DCF regimens have similar efficacy and a similar tolerability profile for first line treatment of advanced gastric cancer. The decision of the first line chemotherapy in advanced gastric cancer could be improved with patient selection according to clinical parameters and molecular markers.
\end{abstract}

Keywords: Gastric cancer - chemotherapy - TCF protocol - ECF regimen

Asian Pac J Cancer Prev, 15 (16), 6727-6732

\section{Introduction}

Gastric cancer (GC) is the second leading cause of cancer death and the fourth most common type of cancer. Globally, 989,600 new cases and 738,000 deaths per year can be encountered (Nagini, 2012). Major risk factors for stomach cancer are hypothesized to be nutritional and environmental, including Helicobacter pylori (H.pylori) infection, the prevalence of which ranges from $25 \%$ in developed countries to $80-90 \%$ in developing countries (Paunder, 2002). The geographic distribution of GC is characterized by a wide international variation; high-risk areas include East Asia, Eastern Europe, and parts of Central and South America. The incidence in the Middle East countries is relatively low, with rates 5-15 times lower than in Japan (Parkin, 2002). In the treatment of unresectable or metastatic gastric cancer, chemotherapy leads to a significant survival difference compared to best supportive care (BSC), and could relieve gastric cancer-related symptoms, and improve quality of life (Kucukzeybek et al., 2012).

Many drugs are active in the first line chemotherapy of AGC like flouropyrimidines, platinium derives, epirubicin, taxenes and irinotecan. Although the array of chemotherapy agents available for treating GC is increasing, no consensus has emerged regarding optimal palliative chemotherapy for advanced disease. Recently in FISH positive patients, anti-HER 2 combined therapies prefered to use after TOGA trial, but in FISH negative patients its stil contoversial to determine the chemotherapy regimen (Bang et al., 2010).

\section{Materials and Methods}

\section{Study design}

This was a single center retrospective study. The hospital records of patients who had received systemic chemotherapy for gastric malignancy between 2006 and 2013 were retrospectively investigated. Onehundered and eight patients were treated with either DCF (Docetaxel, cisplatin and fluorouracil) or ECF (Epirubicin, cisplatin and fluorouracil) regimens. Twenty-one patients discontinued therapy and finally data were collected from 86 cases for the retrospective analysis.

All the data were prospectively recorded. Medical records included physical examination, surgical and pathological reports, imaging records, chemotherapy regimens, response, progression time, toxicity profile, 
final follow-up and time of death. Approval of the study was obtained from the faculty Ethics Committee. Pretreatment evaluation included a determination of medical history, physical examination, complete blood cell (CBC), blood chemistry, gastroscopy, chest radiograph, and computerised tomography (CT) scan of the abdomen. Tumor evaluations were carried out every 2 months until disease progression on the basis of the response evaluation criteria in solid tumors (RECIST) criteria version 1.0. Complete response (CR), partial response (PR), stable disease (SD), and progressive disease (PD) were defined according to the standard World Health Organization criteria. Data from patients who received at least 1 cycle of chemotherapy were included in the safety analysis. Toxicity was graded according to the NCI Common Terminology Criteria for Adverse Events (CTCAE) v 4.03 between 1 to 5 .

The DCF regimen consisted of docetaxel $50-75 \mathrm{mg} / \mathrm{m}^{2}$ IV infusion on $\mathrm{d} 1$, cisplatin $50-75 \mathrm{mg} / \mathrm{m}^{2} \mathrm{IV}$ infusion on $\mathrm{d} 1$, fluorouracil $500-750 \mathrm{mg} / \mathrm{m}^{2}$ as continuous IV infusion for 5 days. The ECF regimen consisted of epirubicin $50 \mathrm{mg} /$ $\mathrm{m}^{2}$ IV infusion on $\mathrm{d} 1$, cisplatin (CDDP) $60 \mathrm{mg} / \mathrm{m}^{2} \mathrm{IV}$ infusion on d1 5 -FU $200 \mathrm{mg} / \mathrm{m}^{2}$ continuous IV infusion for 21 days. Chemotherapy was repeated every 3 weeks. All chemotherapies were given by using a portable pump into the subclavian vein. The treating physicians determined the chemotherapy regimen, as well as the initial dose of chemotherapy drugs according to the patient's ECOG status. Treatment was continued until disease progression or lack of clinical benefit, or toxicity. Toxicities were graded according to the toxic effects that developed during the preceding cycle. All patients received standard premedication consisting of hydration, corticosteroid and antiemetic. The prophylactic use of hematopoietic growth factors was not allowed during treatment, except for patients with febrile neutropenia or where grade 4 myelosuppression developed. Chemotherapy was delayed until neutrophils were recovered $(>1500 / \mu \mathrm{l})$ or platelets reached $>100000 / \mu$ l, or until resolution of any significant non-hematological-toxicity. Filgrastim was used in subsequent cycles in cases of National Cancer Institute- Common Toxicity Criteria grade 4 neutropenia or in cases of febrile neutropenia, which were treated with granulocyte colony-stimulating factor and antibiotics.

The dose of all drugs was reduced by $25 \%$ in subsequent cycles in cases of National Cancer InstituteCommon Toxicity Criteria grade 3-4 mucositis and in cases of poor performance status. After this combination chemotherapy had failed, second line chemotherapy was recommended to all the patients if their performance status was preserved.

\section{Objectives of the study}

The primary objective was overall survival (OS). Progression free survival (PFS), response rate (RR), tolerability and toxicity of the two regimens were measured and restrospectively compared. The starting point of OS and progression-free survival (PFS) was the first day of chemotherapy. The date of disease progression or death from causes from other than gastric cancer was used in calculating PFS. OS was defined as the time between the date of diagnosis for metastatic disease and the date of death from any cause. OS and PFS were calculated by the Kaplan-Meier method with the log-rank test. A p value of $<0.05$ was accepted as significant. Multivariate Cox regression models were used to examine the impact of clinical and treatment variables on the outcomes of chemotherapy.

\section{Statistics}

The comparisons in characteristics and response rate between the patients in the two survivals were evaluated using Kaplan-Meier and Mann-Whitney survival methods. PFS and OS were calculated on the treated population. Multivariate analysis using logistic regression and Cox hazards model were performed to find the correlation between demographic species and response rate and survival.

\section{Results}

\section{Patient characteristics}

Patient characteristics are reported in (Table 1). The ECF and DCF groups were well balanced. The median age was 55 years and more than half of the patients were male. ECOG PS was determined as 0 and 1 in most of the patients. Most of the patients had histologically confirmed adenocarcinoma (93\%); the most frequent histological grade was G2 of the defined differentiation (60\%). Only 4 patients had gastroesophageal junction cancer $(4.6 \%)$. Seventy-three patients had metastatic disease $(85 \%)$ and 13 patients had locally advanced disease $(15 \%)$. The most common sites of metastases were the peritoneum and the liver. Approximately one third of the patients had two or more metastatic disease sites mostly involving the liver and peritoneum. Blood group 'A' was significantly higher than other groups. The median body mass index (BMI) was 23.3.

\section{Chemotherapy characteristics}

A total of 405 chemotherapy courses were administered. The median number of course received was 6 . Of the total 108 patients, 22 could not be evaluated for responses because of early discontinuation of therapy. A total of 42

\section{Table 1. Patient Characteristics}

\begin{tabular}{|c|c|c|c|c|c|}
\hline & & $\begin{array}{l}\text { Tot } \\
(\%\end{array}$ & $\begin{array}{c}\mathrm{ECF} \\
(\%)\end{array}$ & $\begin{array}{c}\mathrm{DCF} \\
(\%)\end{array}$ & $\mathrm{p}$ \\
\hline \multirow[t]{2}{*}{ Gender } & Male & 61 & 64 & 50 & \\
\hline & Female & 39 & 36 & 50 & 0.29 \\
\hline Age & Median (Range) & 55 & $57(30-77)$ & $54(25-72)$ & 0.11 \\
\hline \multirow[t]{2}{*}{ ECOG } & 0 & 32.5 & 27.3 & 38.1 & \\
\hline & 1 & 51.1 & 50 & 52.4 & \\
\hline \multicolumn{6}{|c|}{ Site of metastasis } \\
\hline & Liver & 59 & 65 & 45 & \\
\hline & peritoneum/LAP & 62 & 62 & 59 & \\
\hline & Lung & 17 & 15 & 11 & \\
\hline & Ovarium & 20 & 18 & 18 & \\
\hline \multirow[t]{2}{*}{ Histology } & Adenocarcinoma & 89 & 93 & 92.5 & 1 \\
\hline & Non-adenocarcinoma & 10 & 7 & 7.5 & \\
\hline \multirow[t]{2}{*}{ Blood group } & A & 59 & 71.1 & 66.7 & 0.31 \\
\hline & Other & 41 & 28.9 & 33.3 & \\
\hline
\end{tabular}

*Abbreviations: ECOG=Eastern Cooperative Oncology Group; GEJ=gastroesophageal junction, LAP=lymphadenopathy 
patients $(48 \%)$ received the DCF regimen, 44 patients received the ECF regimen (52\%) (Table 2). After failure of first line therapy, second line chemotherapy was administered to more than one third of the patients $(n=36)$ specifically cisplatin and capecitabine $(52 \%)$ was administered as second line in both groups.

\section{Efficacy and Survival}

A median of 6 cycles (range, 1-9) per patient were administered. Twenty six patients $(30 \%)$ received fewer than the planned 6 because of toxicity or early progression (3 early deaths from toxicity). If there was tumor regression and the patient was deemed fit, 2 or 3 cycles were added to the 6 cycles. Table 2 lists the overall tumor response rates (ORR). The objective response rate in the ECF group was $29.5 \%$ (4.5\% CR and $25 \% \mathrm{PR}$ ) versus $26.2 \%$ (2.4 CR and $23.8 \% \mathrm{PR}$ ) in the DCF group

Table 2. Overall Objective Response Rates for First Line Chemotherapy Of Gastric Cancers

\begin{tabular}{lcc}
\hline Response & ECF\% & DCF\% \\
\hline CR & 4.5 & 2.4 \\
PR & 25 & 23.8 \\
ORR (CR+PR) & 29.5 & 26.2 \\
SD & 15.9 & 23.8 \\
PD & 52.3 & 45.2 \\
Early death or severe toxicity & 2.2 & 4.7 \\
\hline
\end{tabular}

*Abbreviations: $\mathrm{CR}=$ complete response; $\mathrm{DCF}=$ docetaxel, cislatin and fluorouracil $\mathrm{ECF}=$ epirubicin, cisplatin and fluorouracil; $\mathrm{ORR}=$ overall response rate; $\mathrm{PD}=$ progressive disease; $\mathrm{PR}=$ partial response; $\mathrm{SD}=$ stable disease

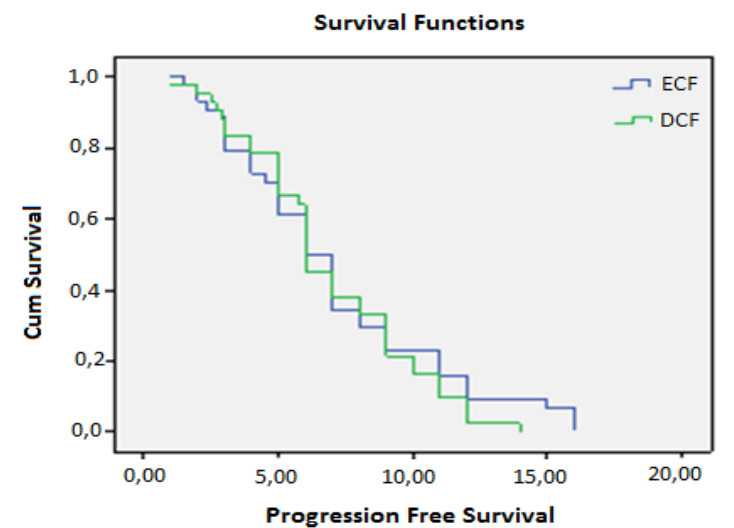

Figure 1. Kaplan-Meier Estimate of Progression Free Survival with ECF or DCF

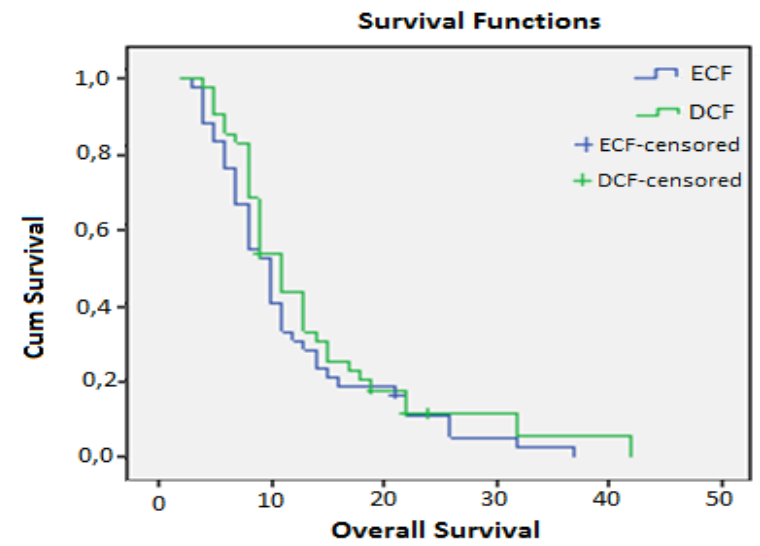

Figure 2. Kaplan-Meier Estimate of Overall Survival with ECF or DCF
Table 3. Hematological Toxicity

\begin{tabular}{lcccc}
\hline & \multicolumn{2}{c}{ ECF\% $(\mathrm{n}=44)$} & \multicolumn{2}{c}{ DCF\% $(\mathrm{n}=42)$} \\
& All & Grade & All & Grade \\
& grades & $3-4$ & grades & $3-4$ \\
\hline Anemia & 47.7 & 13.6 & 45.2 & 7.1 \\
Leukopenia & 43.2 & 13.6 & 23.8 & 16.7 \\
Neutropenia & 52.3 & 9.1 & 23.8 & 16.7 \\
Thrombocytopenia & 6.8 & 2.3 & 2.4 & - \\
\hline
\end{tabular}

Table 4. Non-Hematological Toxicity

\begin{tabular}{lcccc}
\hline & \multicolumn{2}{c}{ ECF\% $(\mathrm{n}=44)$} & \multicolumn{2}{c}{ DCF\% $(\mathrm{n}=42)$} \\
& All & Grade & All & Grade \\
& grades & $3-4$ & grades & $3-4$ \\
\hline Anorexia & 47.7 & 9.1 & 57.1 & 2.4 \\
Nause-Vomiting & 50 & 6.8 & 52.4 & 4.8 \\
Fatigue & 63.6 & 4.5 & 54.8 & 7.1 \\
Stomatitis & 6.8 & - & - & - \\
Diarrea & 4.5 & - & - & - \\
Trombosis & 11.4 & 2 & 2.4 & 4.8 \\
Hepatotoxicity & 11.4 & - & 9.4 & - \\
Nephrotoxicity & 2.3 & - & 9.4 & - \\
\hline
\end{tabular}

( $p>0.05$ ). Stable disease rates were $16.3 \%$ and $25.0 \%$ respectively $(\mathrm{p}>0.05)$.

The median PFS for ECF and DCF was 6.0 months (95\% confidence interval $(\mathrm{CI}), 4.9-7.0$ for ECF and 5.36.6 for DCF respectively, log-rank=0.48) (Figure 1). Survival data were assesed for all treated patients and 81 of 86 patients (94\%) died. The overall survival (OS) was 10.0 months $(95 \%$ confidence interval $(\mathrm{CI}, 8.2-11.7)$ in the ECF group and 11.0 months (95\% confidence interval (CI), 9.1-12.8) in the DCF group (log-rank $p=0.31$ ) (Figure 2). In particular DCF patients had a longer PFS than ECF patients. However, no statistically significant difference was found between each regimen used as first line CT. Second line CT was administered $42 \%$ in the ECF and $45 \%$ in the DCF groups respectively. OS was longer in the group able to receive second line CT than in those without further treatment. Multivariate analysis showed that patient characteristics including gender, site of primary tumor and histology had no effect on survival or response to chemotherapy.

\section{Safety}

Overall, ciplatin based combination chemotherapies were generally well tolerated. Most of the patients were treated at full doses without dose reduction or any delay. The main cause of non-compliance was hematological toxicity. The incidence and severity of adverse events, irrespective of grade, were fatigue, anorexia, nauseavomiting, leukopenia and neutropenia in both groups. The majority of hematological adverse events (Table 3) and non-hematological adverse events (Table 4) were grade $1 / 2$. The most frequent grade $3 / 4$ adverse events in both groups were anorexia, fatigue, vomiting and neutropenia. No sigificant difference was found in the occurrence of grade $3 / 4$ toxicities between regimens. Three possible treatment-related deaths were identified. Death occured in 1 patient while receiving $\mathrm{CT}$ and 2 deaths were attributed to neutropenic sepsis. 


\section{Discussion}

The epidemiology of GC has been widely studied in the west as well as in Japan (Matsuda, 2013). Recent studies showed the polymorphisms of dihydropyrimidine dehydrogenase genes and tymidylate synthase genotips are valuable predictors of the response to fluorouracil based chemotherapy for gastric cancer patients in adjuvant and metastatic setting (Zhang et al., 2012, Gao et al., 2013). Tumor biology may differ between patients in western countries and the eastern countries. However, few reports from developing countries have been published. This lack of data can affect the treatment modalities. For example, $\mathrm{S} 1$ plus cisplatin is accepted as standard first line CT for advanced gastric cancer in Japan whereas $\mathrm{S} 1$ is not popular in western countries. Good descriptive data on western countries are lacking. The current study showed some epidemiological differences. For example, the median age at presentation was 55.1 years in the current study and it was correlated with another study from same region (Selcukbiricik, 2013). But the median age in the United States is 70 years for men and 74 years for woman (Nomura, 1996). This can be explained by a lower socioeconomic status and higher prevalence of $\mathrm{H}$. Pylori infection in Turkey and Middle East than United States and Western Europe (Ozaydin et al., 2013). H.pylori infection has been generally accepted as an important factor in gastric carcinogenesis and in China genetic properties (rs1550117 polimorphism) increases the risk for H.Pylori infection (Cao et al., 2013). High rates of h.pylori may cause gastric cancer in younger ages in some regions of the world but it should be noted that h.pylori is not a single risk factor, eating habits and genetic disorders are also very important factors.

The current study showed that stomach was the most common site (95\%) for gastric cancer. Gastroesophageal junction tumors are still of very low incidence. In the United States there is a rising trend in gastric cardia tumors, including lower esophageal and GEJ tumors. But it should be noted that our study group was only consist of metastatic and unresectable patients and junction tumors are usually early diagnosed owing to the severe dysphagia and may have surgery at the early stage.

AGC is an incurable condition where the aim of treatment is to improve survival and to palliate symptoms. Wagner et al. (2010) published a meta-analysis of three trials comparing chemotherapy with BSC, and they found a significant benefit in OS in favor of chemotherapy and revealed that there was an improvement in median survival from 4.3 to 11 months. The best choice of chemotherapy regimens for patients with AGC is still a matter of controversy and requires further investigation. Despite recent advances in the treatment of AGC, patients treated with first line chemotherapy have median OS rarely exceeding 12 months (Wagner et al., 2006). Currently, fluoropyrimidine and cisplatin combination chemotherapy is accepted as a standard regimen. It is presently unclear whether the triplet combination is superior to cisplatinbased doublets for patients with AGC. A previously conducted meta-analysis showed a difference in OS of approximately 2 months in favor of the anthracycline- containing triplets versus doublets. Docetaxel-containing triplet chemotherapy (docetaxel, cisplatin and 5-FU) showed superior time-to-progression that of doublet (cisplatin and 5-FU). From the data available so far, the use, in patients with a good performance status, of a three-drug combination chemotherapy seems feasible. Randomized Phase II (Roth et al., 2007) and phase III studies (Van Cutsem et al., 2006) on triplets as ECF (epirubicin, cisplatin, and 5-FU) and TCF (docetaxel), have shown an increase in response rate from $35 \%$ to $40 \%$ with a median survival estimated between 8 and 11 months. Although ECF would be regarded as the standard of care in the United Kingdom, this regimen has not been widely accepted in North America. Despite the availability of numerous effective drugs and different combinations, it is still not possible to define a recommended chemotherapy regimen in patients with gastric cancer that is HER2 negative or with unknown receptor status. In the current study; Her-2 status of the patients is unknown at most of the patients. Another study in FISH positive gastric cancer patients receiving trastuzumab is ongoing in our department. This study compared two most popular regimens in first line treatment of AGC and showed no significant difference in RR, PFS and overall survival. In the current study, median OS was 10 months in the ECF group and 11 months in the DCF group. In a study from China, Gao et al showed median OS was 10,8 months with DCF and 8,06 months with non-taxane containing regimens (Gao et al., 2010). In another trial with docetaxel, oxaliplatin and capecitabine combination PFS and OS was 6,9 and 12,6 months respectively. The most common grade 3-4 toxicity was neutropenia (41\%) in that trial wheras 9,1 and 16,7 in ECF and DCF group respectively in our study (Louro et al., 2013). Yeh et al showed that FOLFOX has 11,2 months median OS in first line setting in advanced or recurrent gastric cancer (Yeh et al., 2012). The result of our study was correlated with literature.

It could be some potential factors associated with survival, one of them is the median ages of two groups. They were similar in both of two groups. The other factor was histology and more than ninety percent of histology was adenocarcinoma in both groups. We knew that number of metastatic sites had prognostic factor on survival. This was a retrospective study and all properties of the patients may not be distributed equally. When we analysed it we noticed that the liver involvement were slightly more in ECF group this may have led worse survival rates. Likewise sum of ECOG 0 and 1 patients were slightly more in DCF group, this situation may affect the survival positively.

In the current study median course of chemotherapy was six. The role of maintenance therapy except biologic agents are controversial. We usually do not prefer maintenance therapy in our clinic and the result of median six courses show that both regimens were well tolerated and most of the patients completed the planned courses. The main toxicity was hematological in both groups. Grade $1 / 2$ toxicities were slightly more in ECF group but there was no statistically difference in grade $3 / 4$ toxicities. Only two deaths were attributed to the neutropenic sepsis. According to national comprehensive cancer 
network (NCCN) DCF is a high risk regimen and ECF is an intermediate risk regimen for febrile neutropenia. In high risk rejimens profilactic colony stimulating factor (CSF) is suggested at category 1 level. We didn'use CSF in all patients as a primary prophylaxis unless febrile neutropenia occurred during courses. Nearly half of the DCF group has received modified DCF regimen and no CSF prophylaxis required. Neutropenia rate was lower than the other similar studies. But it should be noted that drug doses were higher in that trials (Docetaxel dose up to $300 \mathrm{mg} / \mathrm{m}^{2}$ and cisplatin dose upto $360 \mathrm{mg} / \mathrm{m}^{2}$ ). The other most observed toxicities are fatigue, anorexia and vomiting. These results were correlated with the literature but stomatitis and diarrohea were lower than literature. The anaphylaxis were seen in 11,8\% patients in that trials but in our study any anaphylaxis were seen in the docetaxel group (Chen et al., 2013). We use dexametazone two days before docetaxel for prophylaxis standartly in our clinic and the anaphylaxis rates are lower than the expected. Although cisplatin is often used in combination with other agents, it is well known that cisplatin is associated with significant toxicity and usually requires a high level of clinical monitoring and supportive care including intensive intravenous hydration. Adverse events were similar and most of them were grade 1-2.

There are some limitations in this study. This was a retrospective study but there was no bias in the regimen selection. The major limitation of this study is that it was a single center study with a small number of patients. The study included patients who were evaluated and treated at a single institution, which may not reflect the whole Turkish population, but it may show the Northeast region characteristics. The results of response rate and PFS are similar to those of other trials of advanced gastric cancer. The comparisons of OS among different clinical trials is limited because of the heterogenity of patient populations, baseline variables such as patient age, PS, and extent of the disease, the use of second-line chemotherapy and the population analyzed. The advantage of the study was that it was carried out in a caucasion only (Turkish) population, which probably narrows down the diversity of the response. In the current analysis, there was no advantage of one cisplatin based triplet over the other. While there was no relevant difference in OS between treatment groups, second line therapy could have had an effect on survival. More than one third of the patients received second line chemotherapy. Most of those were doublet or single agent chemotherapies. Very few patients received three or further lines due to poor performance status and short survival. Oxaliplatin-based regimens have been actively investigated to improve the efficacy and tolerability of combination chemotherapy for AGC patients. Oxaliplatin has significant activity against some cisplatin-resistant tumors and a favorable safety profile over cisplatin (Tozowa et al., 2008). The quality of life (QoL) may also affect the treatment decision. Different trials have investigated QoL in advanced gastric cancer. Ajani et al showed that DCF achieved better preservation of QOL compared with CF (Ajani et al., 2007). Another limitation of the current study is that QoL was not evaluated because the study was retrospective.
Although doublet combinations are standart in many oncology clinics, triplet combination with antracycline or taxane seems feasible without increasing the toxicity. In summary, both ECF and DCF regimens have similar efficacy and a similar tolerability profile for first line treatment of advanced gastric cancer and both are considered reasonable second-line treatment options. The taxane trials include QoL analysis but it was not compared with another triplet combination prospectively. Until trastuzumab and/or ramicirumab replace of the first line therapy in advanced gastric cancer it could be improved patient selection of chemotherapy combinations according to clinical parameters and molecular markers.

\section{Acknowledgements}

The statistical analysis of this study has been supported by our colleague Yusuf Güney from Public Health Department.

\section{References}

Ajani JA, Moiseyenko VM, Tjulandin S, et al (2007). Quality of life with docetaxel plus cisplatin and fluorouracil compared with cisplatin and fluorouracil from a phase III trial for advanced gastric or gastroesophageal adenocarcinoma: the V-325 Study Group. J Clin Oncol, 25, 3210-16.

Bang YJ, Van Cutsem E, Feyereislova A et al (2010). Trastuzumab in combination with chemotherapy versus chemotherapy alone for treatment of HER2-positive advanced gastric or gastro-oesophageal junction cancer (ToGA): a phase 3 , open-label, randomised controlled trial. Lancet, 28, 687-97.

Cao X-Y, Jia Z-F, Cao D-H, et al (2013). DNMT3a rs1550117 Polymorphism association with increased risk of Helicobacter pylori infection. Asian Pac J Cancer Prev, 14, 5713-18.

Chen X-L, Chen X-Z, Yang C, et al (2013). Docetaxel, cisplatin and fluorouracil (DCF) regimen compared with non-taxanecontaining palliative chemotherapy for gastric carcinoma: a systematic review and meta-analysis. PLOS ONE, 8, 603320

Di Lauro L1, Vici P, Belli F et al (2013). Docetaxel, oxaliplatin, and capecitabine combination chemotherapy for metastatic gastric cancer. Gastric Cancer. Dec 8. [Epub ahead of print].

Gao J, He Q, Hua D et al (2013). Polymorphism of TS 3'-UTR predicts survival of Chinese advanced gastric cancer patients receiving first-line capecitabine plus paclitaxel. Clin Transl Oncol, 15, 619-25.

Gao H, Ding X, Wei D et al (2010). Docetaxel versus epirubic in combined with cisplatin, leucovorin and fluorouracil for advanced gastric carcinoma as first line therapy: a randomized clinical trial. Chin J Clin Oncol, 15, 529-533.

Kucukzeybek Y, Dirican A, Erten C, et al (2012). Second-line irinotecan after cisplatin, fluoropyrimidin and docetaxel for chemotherapy of metastatic gastric cancer. Asian Pac J Cancer Prev, 13, 2771-4.

Luigi Di Lauro, Patrizia Vici, Franca Belli et al (2013). Docetaxel, oxaliplatin, and capecitabine combination chemotherapy for metastatic gastric cancer. Gastric Cancer, Dec 8, [Epub ahead of print].

Matsuda T, Saika K (2013). The 5-year relative survival rate of stomach cancer in the USA, Europe and Japan. Jpn J Clin, 43, 1157-58

Nagini S (2012). Carcinoma of the stomach: a review of epidemiology, pathogenesis, molecular genetics and chemoprevention. World J Gastrointest Oncol, 4, 156-69.

Nomura A. Stomach cancer, in Schottenfeld D (1996). Cancer 
Fatih Teker et al

epidemiology and prevention 2nd ed. Oxford University Press, pp. 707-24.

Ozaydin N, Turkyilmaz SA, Cali S (2013). Prevalence and risk factors of helicobacter pylori in Turkey: a nationallyrepresentative, cross-sectional, screening with the ${ }^{13} \mathrm{C}$-Urea breath test. BMC Public Health, 13, 1215.

Paunder RE (1995). The prevention of H. pylori in different countries. Aliment Pharmacol Ther, 9, 33-40.

Parkin DM (2002). Global cancer statistics. CA Cancer J Clin, 55, 74-108.

Roth AD1, Fazio N, Stupp R et al (2007). Docetaxel, cisplatin, and fluorouracil; docetaxel and cisplatin; and epirubicin, cisplatin, and fluorouracil as systemic treatment for advanced gastric carcinoma: a randomized phase II trial of the Swiss group for clinical cancer research. J Clin Oncol, 25, 3217-23.

Selcukbiricik F, Tural D, Bilici A et al (2013). Clinicopathological features and localization of gastric cancers and their effects on survival in Turkey. Asian Pac J Cancer Prev, 14, 553-6.

Tozawa K, Oshima T, Kobayashi T et al (2008). Oxaliplatin in treatment of the cisplatin resistant MKN45 cell line of gastric cancer. Anticancer Res, 28, 2087-92.

Van Cutsem E, MoiseyenkoVM, Tjulandin S, et al (2006). Phase III study of docetaxel and cisplatin plus fluorouracil compared with cisplatin and fluorouracil as first-line therapy for advanced gastric cancer: a report of the V325 Study Group. J Clin Oncol, 24, 4991-7.

Wagner AD, Unverzagt S, Grothe W, et al (2010). Chemotherapy for advanced gastric cancer. Cochrane Database Syst Rev, [Epub ahead of print].

Wagner AD, Grothe W, Haerting et al (2006). Chemoth advanced gastric cancer: a systematic review and meta-analysis based on aggregate data. J Clin Oncol, 24, 2903-9.

Yeh YS1, Tsai HL, Ma CJ et al (2012). A retrospective study of the safety and efficacy of a first-line treatment with modified FOLFOX-4 in unresectable advanced or recurrent gastric cancer patients. Chemotherapy, 58, 411-8.

Zhang XP1, Bai ZB, Chen BA et al (2012). Polymorphisms of dihydropyrimidine dehydrogenase gene and clinical outcomes of gastric cancerpatients treated with fluorouracilbased adjuvant chemotherapy in Chinese population. Chin Med J, 125, 741-6. 